\title{
Synergistic Interaction of Dietary Pattern and Concordance Lifestyle with Abnormal Liver Function among Young Adults in Taiwan: A Population-Based Longitudinal Study
}

\author{
Rathi Paramastri ${ }^{1} \mathbb{D}$, Chien-Yeh Hsu ${ }^{2,3,+}$, Yung-Kun Chuang ${ }^{4,5,6,+} \mathbb{D}$, Hsiu-An Lee ${ }^{7}$, Bayu Satria Wiratama ${ }^{8} \mathbb{D}$ \\ and Jane C.-J. Chao ${ }^{1,3,6, *(D)}$
}

1 School of Nutrition and Health Sciences, College of Nutrition, Taipei Medical University, 250 Wu-Hsing Street, Taipei 11031, Taiwan; rara.paramastri@gmail.com

2 Department of Information Management, National Taipei University of Nursing and Health Sciences, 365 Ming-Te Road, Peitou District, Taipei 11219, Taiwan; cyhsu@ntunhs.edu.tw

3 Master Program in Global Health and Development, College of Public Health, Taipei Medical University, $250 \mathrm{Wu}$-Hsing Street, Taipei 11031, Taiwan

4 Master Program in Food Safety, College of Nutrition, Taipei Medical University, 250 Wu-Hsing Street, Taipei 11031, Taiwan; ykchuang@tmu.edu.tw

5 School of Food Safety, College of Nutrition, Taipei Medical University, 250 Wu-Hsing Street, Taipei 11031, Taiwan

6 Nutrition Research Center, Taipei Medical University Hospital, 252 Wu-Hsing Street, Taipei 11031, Taiwan

7 National Health Research Institutes, 35 Keyan Road, Zhunan Town, Miaoli 350, Taiwan; billy72325@gmail.com

check for

updates

Citation: Paramastri, R.; Hsu, C.-Y.; Chuang, Y.-K.; Lee, H.-A.; Wiratama, B.S.; Chao, J.C.-J. Synergistic

Interaction of Dietary Pattern and

Concordance Lifestyle with

Abnormal Liver Function among

Young Adults in Taiwan: A

Population-Based Longitudinal Study.

Nutrients 2021, 13, 3591.

https://doi.org/10.3390/nu13103591

Academic Editor: Naoki Tanaka

Received: 30 August 2021

Accepted: 12 October 2021

Published: 14 October 2021

Publisher's Note: MDPI stays neutral with regard to jurisdictional claims in published maps and institutional affiliations.

Copyright: (c) 2021 by the authors. Licensee MDPI, Basel, Switzerland. This article is an open access article distributed under the terms and conditions of the Creative Commons Attribution (CC BY) license (https:/ / creativecommons.org/licenses/by/ $4.0 /)$.
8 Department of Epidemiology and Biostatistics, Faculty of Medicine Public Health and Nursing, Universitas Gadjah Mada, Yogyakarta 55281, Indonesia; bayu.satria@ugm.ac.id

* Correspondence: chenjui@tmu.edu.tw; Tel.: +886-2-2736-1661 (ext. 6548); Fax: +886-2-2737-3112

+ These authors contributed equally to this work.

Abstract: While diet and lifestyle are independently implicated in the etiology of liver disease, the interaction of diet and lifestyle may be more helpful for determining the risk of liver abnormality. Thus, our study aimed to evaluate the interaction between the dietary pattern associated with liver biomarkers and lifestyle factors among Taiwanese adults with abnormal liver enzymes. A liverassociated dietary pattern, generated using reduced rank regression, was characterized by high intake of soy sauce or other dips, sugar sweetened beverages, and preserved and processed foods, but low intake of seafood, fruits, eggs, and dark-colored vegetables. In the fully adjusted model, liver-associated dietary patterns or unhealthy concordance lifestyle factors were associated with an increased risk of having liver function abnormality (OR $=1.08,95 \% \mathrm{CI}: 1.04,1.12$ and $\mathrm{OR}=1.42$, 95\% CI: 1.31, 1.53, respectively). Moreover, the interaction between liver-associated dietary pattern and unhealthy concordance lifestyle factors showed more significant correlation, with an elevated risk of abnormal liver function ( $\mathrm{OR}=2.14,95 \% \mathrm{CI}: 2.02,2.26)$. Therefore, our study suggests that participants who have a strong liver-associated dietary pattern along with unhealthy concordance lifestyles are likely to have increased odds of abnormal liver function.

Keywords: dietary pattern; smoking; alcohol drinking; sleep; physical activity; body mass index; liver function; adults; reduced rank regression

\section{Introduction}

Liver disease represents the hepatic manifestation of complex metabolic symptoms [1] and often coexists with obesity, dyslipidemia, and insulin resistance [2]. Evaluating the hepatic dysfunction includes the diagnosis of hepatocyte injury and structural liver disease based on signs, symptoms, and biochemical measures of serum biomarkers $[3,4]$. Serum hepatic enzymes such as alanine transaminase (ALT), aspartate transaminase (AST), and gamma-glutamyltransferase $(\gamma-\mathrm{GT})$ have been used as parameters for diagnosing defects 
in liver function [5]. Several conditions of damaged liver can be identified by the elevation of serum ALT, AST, and $\gamma$-GT [6,7].

The emerging liver disorder commonly found in adults is nonalcoholic fatty liver disease (NAFLD). Globally, the estimated prevalence of NAFLD is $24 \%$ in the general population, with the highest number acquired from South America and the Middle East, followed by Asia and Europe [8]. Among individuals aged $\geq 20$ years, the prevalence of NAFLD ranged from $20-30 \%$ in the Hong Kong, South Korea, and Taiwan populations in 2015 [9]. A previous study using Markov modelling to project disease burden reported that the prevalence of NAFLD was estimated to rise from 6 to $20 \%$ between 2019 and 2030; likewise, the mortality rate of NAFLD was also projected to increase from 65 to $100 \%$ [9]. The previous study suggested that increased incidence of NAFLD in the AsiaPacific region was ascribed to the latest adaptation of Westernized dietary and lifestyle habits [9]. Correspondingly, unhealthy dietary habits and sedentary lifestyle have been adopted rapidly in a habitual lifestyle among Taiwan's population, and the risk of chronic disease is expected to increase [10].

Dietary habits have been considered as a potential environmental factor in relation to liver disease. A study among adults in Taiwan showed that individuals in the highest quartile of a Western dietary pattern, characterized by animal protein, eggs, refined foods and beverages, oil-cooked foods, and high-sugar foods, were likely to have elevated ALT levels (OR $=1.43,95 \%$ CI: 1.04, 1.97), while individuals consuming the Mediterranean diet were correlated with a reduced risk of having abnormal levels of $\gamma$-GT (OR $=0.72$, $95 \%$ CI: $0.53,0.97$ [11]. In accordance, a previous investigation among Iranian adults found greater odds of having NAFLD in subjects with the highest adherence to a Western dietary pattern (OR $=2.61,95 \%$ CI: $1.41,4.28)$; meanwhile, subjects in the highest quartile of a healthy dietary pattern were $41 \%$ less likely to have NAFLD after controlling for confounders [12]. Moreover, a previous report also mentioned that the role of sedentary lifestyle or physical inactivity was independent from the progression of liver disease [13]. A review investigation of 20 observational studies revealed that the pooled odds ratio for the association between actively smoking and NAFLD was 1.11 (95\% CI: 1.03, 1.19) [14]. Therefore, both dietary habits and lifestyles were suggested to be potentially associated with liver dysfunction.

Despite investigations evaluating the roles of dietary habits and lifestyle variables in chronic disease progression, very few studies have aimed to explore the association between them. Because dietary habits and lifestyles cannot be separated from one another in terms of daily activities, such an investigation is crucial for understanding the interaction of dietary habits and lifestyles on liver dysfunction. Many studies have focused on related aspects, such as nutrition and physical activity, while neglecting other modes of social behavior. Thus, our objective was to investigate the interaction between dietary patterns associated with liver biomarkers and lifestyle factors associated with abnormal serum liver enzymes among Taiwanese adults in order to take a step toward preventing liver disease. Moreover, to the best of our knowledge, our study is the first one to investigate the synergistic effect of dietary patterns and lifestyle habits on liver dysfunction in Taiwan.

\section{Materials and Methods}

\subsection{Study Cohort and Design}

Our study was a longitudinal study using data from Mei Jau (MJ) database, which contained data from a prospective study of Taiwanese populations who participated in multiple comprehensive health screening examinations. Prior to enrollment, all participants had read, understood, and signed the informed consent forms provided by MJ Health Management Institution; the study collected no personal identification and information was used for research purposes only. Information on MJ Health Screening Centers has been clearly defined in previous studies $[15,16]$. Participants completed a self-reported questionnaire related to sociodemographic data, lifestyle habits, history of chronic diseases, and dietary consumption at baseline. Participants also underwent physical examination 
and provided biological samples for laboratory testing in accordance with ISO 9001 quality management standards.

Initially, we retrieved 190,200 participants from the MJ database from 2001 to 2015. From this, we randomly recruited participants who met the following criteria: (1) aged between 20 and 45 years, (2) free of chronic diseases, (3) having normal levels of serum liver enzymes at baseline and no history of receiving hepatic treatment, (4) having at least one follow-up visit, and (5) having complete data at baseline. Finally, we included 62,645 participants for analysis. The study design was approved by Taipei Medical University-Joint Institutional Review Board (TMU-JIRB N202001055).

\subsection{Socio-Demographic Characteristics and Lifestyle Factors}

Information related to socio-demographic characteristics and lifestyle factors was collected using a self-reported questionnaire or obtained via face-to-face interviews by trained medical professionals. The collected socio-demographic information included age, gender, marital status, education, and family income. Lifestyle factors, including smoking status, alcohol drinking, sleep habits, and physical activity, were divided into certain categories, which were clearly described in a previous study [17]. We categorized each lifestyle factor into quartiles, and the higher quartile represented the higher degree of unhealthy lifestyle. Moreover, we combined smoking status, drinking status, sleep duration, and physical activity as one group of concordance lifestyle, which was also divided into quartiles to indicate the degree of unhealthy lifestyle.

\subsection{Anthropometric, Biochemical, and Clinical Data}

The assessment of anthropometric measurements, including body weight $(\mathrm{kg})$, height $(\mathrm{cm})$, and body fat (\%), was performed in MJ Health Screening Centers. Body weight and height were assessed using electronic scales and an auto-anthropometer (KN-5000A, Nakamura, Tokyo, Japan). Body mass index (BMI) was calculated as weight $(\mathrm{kg})$ divided by the square of height $\left(\mathrm{m}^{2}\right)$ and further classified into underweight (BMI $<18.5 \mathrm{~kg} / \mathrm{m}^{2}$ ), normal weight $\left(18.5 \mathrm{~kg} / \mathrm{m}^{2} \leq \mathrm{BMI}<24 \mathrm{~kg} / \mathrm{m}^{2}\right)$, overweight $\left(24 \mathrm{~kg} / \mathrm{m}^{2} \leq \mathrm{BMI}<27 \mathrm{~kg} / \mathrm{m}^{2}\right)$, and obese (BMI $\geq 27 \mathrm{~kg} / \mathrm{m}^{2}$ ) categories according to Taiwan's criteria [18]. The body fat percentage of participants was measured using a bioelectrical impedance analysis instrument (InBody Co., Ltd., Seoul, Korea).

All blood samples and blood pressure were taken at MJ Health Screening Centers after 12-14 h overnight fasting. The MJ laboratory was responsible for all measurements of blood sample tests. Because we did not obtain further diagnostic information on liver disease, such as the infection of hepatotoxic viruses, liver biopsy, or liver echogenicity, from the MJ database, and serum hepatic enzymes such as ALT, AST, and $\gamma$-GT are frequently determined in most health screening facilities and hospitals in Taiwan, we used the enzymatic methodology to evaluate liver function. Our study applied the identification of abnormal liver function based on the abnormal ranges of liver enzymes offered by the MJ laboratory as follows: ALT $>33 \mathrm{U} / \mathrm{L}$, AST $>27 \mathrm{U} / \mathrm{L}$, or $\gamma$-GT $\geq 39 \mathrm{U} / \mathrm{L}$ for women and $\gamma$-GT $\geq 50 \mathrm{U} / \mathrm{L}$ for men [11,19]. Our study also retrieved data corresponding to iron biomarkers, such as red blood cells $\left(\mathrm{RBC}, \times 10^{6} / \mu \mathrm{L}\right)$, hemoglobin $(\mathrm{mmol} / \mathrm{L})$, hematocrit $(\%)$, mean corpuscular volume (MCV, $\mathrm{fL})$, mean corpuscular hemoglobin $(\mathrm{MCH}, \mathrm{pg})$, mean corpuscular hemoglobin concentration (MCHC, g/dL), iron ( $\mu \mathrm{g} / \mathrm{dL})$, and ferritin $(\mathrm{ng} / \mathrm{mL})$.

\subsection{Dietary Assessment}

Dietary data were obtained using a standardized and validated semi-quantitative food frequency questionnaire (SQ-FFQ) designed by MJ Health Management, which contained 22 food items generated from 85 closed-ended questions on dietary habits $[15,16]$. The frequency of consuming a fixed serving daily or weekly in the previous month prior to data collection was answered by self-reporting. Each food item question had 5 response options for the frequency of consumption, as mentioned previously $[15,20]$. From the dietary data of the SQ-FFQ, we derived the dietary pattern using a reduced rank regression approach, 
which combined a priori and exploratory statistical methods [21]. For generating the dietary pattern associated with liver function, 7 response variables, including ALT, AST, $\gamma$-GT, alkaline phosphatase (ALP), lactate dehydrogenase (LDH), albumin, and total bilirubin, were applied, and 22 food items were used as the predictors. We only retracted food items with absolute factor loadings $\geq 0.20$ to obtain the most representative dietary habits of study participants $[22,23]$. The scores of the derived dietary patterns were classified into quartiles, and the higher quartile of the derived dietary pattern, the higher the risk for abnormal liver enzymes.

\subsection{Statistical Analysis}

The current study described the distribution of demographic characteristics, lifestyles, clinical data, and biochemical measurements at baseline and follow-up. The characteristics of participants were classified as "normal" and "abnormal" liver function. We examined the normality of data using the Kolmogorov-Smirnov test [24]. The chi-square test for categorical data and Wilcoxon rank-sum test for continuous data were performed to compare the differences between normal and abnormal liver function groups. Furthermore, we performed multinomial logistic regression to predict the risk of abnormal liver function, and factors such as age, gender, family income, education, BMI, body fat, blood pressure, and iron biomarkers, including hemoglobin, hematocrit, iron, and ferritin, were controlled in different models. We estimated multivariate analysis with the adjustment of age and gender in model 1. Moreover, we added marital status, education, family income, BMI, body fat, systolic and diastolic pressure, smoking status, drinking status, sleep duration, physical activity, and iron biomarkers in model 2, except for the factors in model 1, for analysis of the association between liver-associated dietary patterns and the risk of abnormal liver function. For investigation of the association between concordance lifestyle and the risk of abnormal liver function, similar factors without lifestyle factors were adjusted in model 2. Additionally, to assess the potential interaction between liver-associated dietary patterns and concordance lifestyle stratified across the quartiles, we conducted a joint/combined analysis of dietary pattern and concordance lifestyle using the Strobe approach [25], where the reference group was participants in the first quartile of dietary pattern or concordance lifestyle scores. Therefore, we generated 16 combinations between dietary pattern and concordance lifestyle to represent the interaction. Our study performed sensitivity analysis using multivariate linear regression to derive the linear relationship between serum liver biomarkers and quartiles of dietary pattern [26]. All analyses were conducted using STATA version 13 (StataCorp LP, College Station, TX, USA). Significant differences were set using $\alpha=5 \%$.

\section{Results}

\subsection{Characteristics of Study Participants}

In the longitudinal observation from 2001 to 2015, 11,506 participants developed abnormal liver function (18.4\%). Our data revealed that after the participants exhibited altered liver enzymes, this condition remained until the last observation. The demographic and lifestyle characteristics of participants at baseline and follow-up, stratified by liver function, are reported in Table 1. At baseline, the majority of participants were female $(78.8 \%)$, married $(62.5 \%)$, non-smokers $(82.9 \%)$, and non-drinkers $(92.4 \%)$, had education equivalent to high school or above (83.6\%), had a family income $<800,000$ NTD $(60.4 \%)$, had a $6-8 \mathrm{~h}$ sleep duration $(71.1 \%)$, and performed physical activity $\leq 2 \mathrm{~h} /$ week $(77.3 \%)$. Furthermore, these characteristics remained after follow-up observation in both groups. In addition, we found significant differences in the mean of the observational period between the two groups. The participants who experienced abnormal liver function had a shorter observational period than those with normal liver function ( $4.1 \pm 2.7$ vs. $4.8 \pm 3.2$ years). 
Table 1. Demographic and lifestyle characteristics of study participants classified by liver function $(n=62,645)^{\text {a }}$.

\begin{tabular}{|c|c|c|c|c|}
\hline \multirow[b]{2}{*}{ Variables } & \multirow{2}{*}{$\begin{array}{c}\text { Baseline }^{\mathrm{b}} \\
\text { Total } \\
(n=62,645)\end{array}$} & \multicolumn{3}{|c|}{ Follow-Up ${ }^{b}$} \\
\hline & & $\begin{array}{l}\text { Normal Liver Function } \\
\quad(n=51,139)\end{array}$ & $\begin{array}{l}\text { Abnormal Liver Function }{ }^{c} \\
\qquad(n=11,506)\end{array}$ & $p$-Value \\
\hline Age (years) & $37.6 \pm 12.6$ & $38.0 \pm 13.5$ & $42.5 \pm 13.4$ & $<0.0001$ \\
\hline Sex & & & & $<0.0001$ \\
\hline Male & $13,189(21.1 \%)$ & $9223(18.0 \%)$ & $3966(34.5 \%)$ & \\
\hline Female & $49,456(78.8 \%)$ & $41,916(82.0 \%)$ & $7540(65.5 \%)$ & \\
\hline Observational period (years) & & $4.8 \pm 3.2$ & $4.1 \pm 2.7$ & $<0.0001$ \\
\hline Marital status & & & & $<0.0001$ \\
\hline No & $23,519(37.5 \%)$ & $19,705(38.5 \%)$ & $3814(33.1 \%)$ & \\
\hline Married & $39,126(62.5 \%)$ & $31,434(61.5 \%)$ & $7692(66.9 \%)$ & \\
\hline Education & & & & $<0.0001$ \\
\hline$<$ High school & $10,277(16.4 \%)$ & $8144(15.9 \%)$ & $2133(18.5 \%)$ & \\
\hline$\geq$ High school & $52,368(83.6 \%)$ & $42,995(84.1 \%)$ & $9373(81.5 \%)$ & \\
\hline Family income & & & & 0.247 \\
\hline$<800,000$ NTD & $41,417(60.4 \%)$ & $33,863(66.2 \%)$ & $7554(65.7 \%)$ & \\
\hline$\geq 800,000 \mathrm{NTD}$ & $21,228(39.6 \%)$ & $17,276(33.8 \%)$ & $3952(34.3 \%)$ & \\
\hline Smoking status & & & & $<0.0001$ \\
\hline Non-smoker & $51,948(82.9 \%)$ & $42,857(83.8 \%)$ & $9091(79.0 \%)$ & \\
\hline Second-hand smoker & $3635(5.8 \%)$ & $2982(5.8 \%)$ & $653(5.7 \%)$ & \\
\hline Past smoker & $1470(2.4 \%)$ & $1147(2.2 \%)$ & $323(2.8 \%)$ & \\
\hline Smoke occasionally & $1339(2.1 \%)$ & $915(1.8 \%)$ & $424(3.7 \%)$ & \\
\hline Smoke daily & $4253(6.8 \%)$ & $3238(6.4 \%)$ & $1015(8.8 \%)$ & \\
\hline Drinking status & & & & $<0.0001$ \\
\hline Non-drinker & $57,871(92.4 \%)$ & $47,591(93.1 \%)$ & $10,280(89.3 \%)$ & \\
\hline Past drinker & $570(0.9 \%)$ & $444(0.9 \%)$ & $126(1.1 \%)$ & \\
\hline 1-2 times/week & $3096(4.9 \%)$ & $2295(4.5 \%)$ & $801(6.9 \%)$ & \\
\hline 3-4 times/week & $768(1.2 \%)$ & $551(1.1 \%)$ & $217(1.9 \%)$ & \\
\hline Drank daily & $340(0.6 \%)$ & $258(0.4 \%)$ & $82(0.8 \%)$ & \\
\hline Sleep duration & & & & $<0.0001$ \\
\hline Short $(<6$ h) & $12,767(20.4 \%)$ & $10,255(20.1 \%)$ & $2512(21.8 \%)$ & \\
\hline Normal (6-8 h) & $44,562(71.1 \%)$ & $36,455(71.3 \%)$ & $8107(70.5 \%)$ & \\
\hline Long (>8 h) & $5316(8.5 \%)$ & $4429(8.6 \%)$ & $887(7.7 \%)$ & \\
\hline Physical activity & & & & $<0.0001$ \\
\hline Less active ( $\leq 2 \mathrm{~h} /$ week) & $48,404(77.3 \%)$ & $40,006(78.2 \%)$ & $8398(72.9 \%)$ & \\
\hline Active (>2 h/week) & $14,241(22.7 \%)$ & $11,133(21.8 \%)$ & $3108(27.1 \%)$ & \\
\hline
\end{tabular}

${ }^{a}$ Continuous data are presented as mean $\pm \mathrm{SD}$, and categorical data are presented as number (percentage). The $p$-value was analyzed using the Mann-Whitney $\mathrm{U}$ test for continuous variables and the chi-square test for categorical variables. ${ }^{\mathrm{b}}$ Percentage was calculated within the same group. ${ }^{c}$ Abnormal liver function was defined as ALT $>33 \mathrm{U} / \mathrm{L}, \mathrm{AST}>27 \mathrm{U} / \mathrm{L}$, or $\gamma$-GT $\geq 39 \mathrm{U} / \mathrm{L}$ for women and $\gamma-\mathrm{GT} \geq 50 \mathrm{U} / \mathrm{L}$ for men.

The clinical and biochemical data of study participants classified by liver function are given in Table 2. Participants with abnormal liver function had higher proportions of overweight $(22.4 \%$ vs. $13.8 \%)$ and obesity $(10.5 \%$ vs. $5.5 \%)$ compared to those with normal liver function. In addition, body fat, blood pressure, all liver function biomarkers, and all iron biomarkers were significantly higher in participants with abnormal liver function.

\subsection{Liver-Associated Dietary Pattern and Liver Function Biomarkers}

A dietary pattern closely related to liver abnormality was identified in this study using a reduced rank regression (RRR) model. We named the dietary pattern "liver-associated dietary pattern" (Table 3). Dipping sauces, sugar-sweetened beverages, and preserved and processed foods were positively associated with liver-associated dietary patterns (factor loading $>0.20$ ). On the contrary, seafood, fruits, eggs, and dark-colored vegetables showed negative correlations with the observed dietary pattern (factor loading $<-0.20$, 
| factor loading $\mid>0.20$ ). The cumulative percentage of variation explained by the observed dietary pattern was $6.87 \%$, and six response variables, including ALT, AST, ALP, LDH, albumin, and total bilirubin, explained $3.7 \%$ of the total variation (data not shown). Our study found significant correlations between the quartiles of dietary patterns and six of seven serum liver function biomarkers, except for $\gamma$-GT, after controlling for age, gender, marital status, education, family income, BMI, body fat, blood pressure, and lifestyle variables (Supplementary Table S1). Positive correlations were observed between liverassociated dietary patterns and the levels of ALT ( $\beta=0.55,95 \%$ CI: $0.42,0.68)$, AST ( $\beta=0.47$, $95 \%$ CI: 0.26, 0.68), ALP ( $\beta=12.76,95 \%$ CI: $11.90,13.62)$, LDH $(\beta=13.59,95 \%$ CI: 12.59 , 14.58), and total bilirubin ( $\beta=0.01,95 \%$ CI: $11.90,0.01,0.02)$. On the other hand, the highest quartile of liver-associated dietary pattern was inversely correlated with albumin levels $(\beta=-0.02,95 \%$ CI: $-0.02,-0.01)$.

Table 2. Clinical and biochemical data of study participants classified by liver function $(n=62,645)^{a}$.

\begin{tabular}{|c|c|c|c|c|}
\hline \multirow[b]{2}{*}{ Variables } & \multirow{2}{*}{$\begin{array}{c}\text { Baseline }^{b} \\
\text { Total } \\
(n=62,645)\end{array}$} & \multicolumn{3}{|c|}{ Follow-Up ${ }^{b}$} \\
\hline & & $\begin{array}{l}\text { Normal Liver Function } \\
\quad(n=51,139)\end{array}$ & $\begin{array}{l}\text { Abnormal Liver Function } \\
{ }_{(n=11,506)}\end{array}$ & $p$-Value \\
\hline $\begin{array}{l}\text { Body mass index } \\
\qquad\left(\mathrm{kg} / \mathrm{m}^{2}\right) \mathrm{d}\end{array}$ & & & & $<0.0001$ \\
\hline Underweight & $8463(13.5 \%)$ & $7658(14.9 \%)$ & $805(6.9 \%)$ & \\
\hline Normal weight & $40,536(64.7 \%)$ & $33,619(65.7 \%)$ & $6917(60.1 \%)$ & \\
\hline Overweight & $9642(15.4 \%)$ & $7070(13.8 \%)$ & $2572(22.3 \%)$ & \\
\hline Obese & $4004(6.4 \%)$ & $2792(5.6 \%)$ & $1212(10.7 \%)$ & \\
\hline Body fat (\%) & $26.6 \pm 6.7$ & $26.6 \pm 6.9$ & $29.6 \pm 7.8$ & $<0.0001$ \\
\hline $\begin{array}{l}\text { Systolic pressure } \\
(\mathrm{mmHg})\end{array}$ & $113.4 \pm 7.4$ & $113.9 \pm 6.9$ & $122.8 \pm 7.6$ & $<0.0001$ \\
\hline $\begin{array}{l}\text { Diastolic pressure } \\
(\mathrm{mmHg})\end{array}$ & $69.4 \pm 1.8$ & $68.0 \pm 1.3$ & $74.8 \pm 1.9$ & $<0.0001$ \\
\hline \multicolumn{5}{|l|}{$\begin{array}{l}\text { Liver function } \\
\text { biomarkers }\end{array}$} \\
\hline $\operatorname{ALT}(\mathrm{U} / \mathrm{L})$ & $24.3 \pm 2.6$ & $17.6 \pm 7.0$ & $50.6 \pm 4.6$ & $<0.0001$ \\
\hline AST (U/L) & $22.3 \pm 4.6$ & $19.3 \pm 4.2$ & $34.0 \pm 8.5$ & $<0.0001$ \\
\hline$\gamma-\mathrm{GT}(\mathrm{U} / \mathrm{L})$ & $21.5 \pm 2.5$ & $14.6 \pm 5.6$ & $48.7 \pm 4.5$ & $<0.0001$ \\
\hline $\operatorname{ALP}(\mathrm{U} / \mathrm{L})$ & $94.27 \pm 6.4$ & $93.07 \pm 6.3$ & $99.0 \pm 4.7$ & $<0.0001$ \\
\hline LDH $(\mathrm{U} / \mathrm{L})$ & $209.4 \pm 7.9$ & $207.5 \pm 7.7$ & $216.9 \pm 8.7$ & $<0.0001$ \\
\hline albumin $(\mathrm{g} / \mathrm{dL})$ & $4.5 \pm 0.2$ & $4.4 \pm 0.2$ & $4.5 \pm 0.3$ & $<0.0001$ \\
\hline Total bilirubin (mg/dL) & $0.8 \pm 0.3$ & $0.8 \pm 0.3$ & $0.9 \pm 0.3$ & $<0.0001$ \\
\hline \multicolumn{5}{|l|}{ Iron biomarkers } \\
\hline $\mathrm{RBC}\left(\times 10^{6} / \mu \mathrm{L}\right)$ & $4.7 \pm 0.5$ & $4.7 \pm 0.4$ & $4.9 \pm 0.4$ & $<0.0001$ \\
\hline Hemoglobin (mmol/L) & $8.6 \pm 0.9$ & $8.5 \pm 0.8$ & $9.0 \pm 0.9$ & $<0.0001$ \\
\hline Hematocrit $(\%)$ & $41.1 \pm 4.1$ & $40.6 \pm 4.0$ & $43.3 \pm 4.2$ & $<0.0001$ \\
\hline $\mathrm{MCV}(\mathrm{fL})$ & $87.9 \pm 6.7$ & $87.7 \pm 6.8$ & $88.6 \pm 6.2$ & $<0.0001$ \\
\hline $\mathrm{MCH}(\mathrm{pg})$ & $29.5 \pm 2.7$ & $29.4 \pm 2.7$ & $29.8 \pm 2.5$ & $<0.0001$ \\
\hline $\mathrm{MCHC}(\mathrm{g} / \mathrm{dL})$ & $33.6 \pm 0.8$ & $33.5 \pm 0.8$ & $33.6 \pm 0.8$ & $<0.0001$ \\
\hline Iron $(\mu \mathrm{g} / \mathrm{dL})$ & $92.9 \pm 3.7$ & $90.8 \pm 3.7$ & $101.4 \pm 3.7$ & $<0.0001$ \\
\hline Ferritin $(\mathrm{ng} / \mathrm{mL})$ & $125.6 \pm 1.1$ & $107.7 \pm 1.4$ & $214.6 \pm 1.6$ & $<0.0001$ \\
\hline
\end{tabular}

ALT: alanine transaminase, AST: aspartate transaminase, $\gamma$-GT: gamma-glutamyltransferase, ALP: alkaline phosphatase, LDH: lactate dehydrogenase, RBC: red blood cells, MCV: mean corpuscular volume, $\mathrm{MCH}$ : mean corpuscular hemoglobin, MCHC: mean corpuscular hemoglobin concentration. ${ }^{a}$ Continuous data are presented as mean $\pm \mathrm{SD}$, and categorical data are presented as number (percentage). The $p$-value was analyzed between the participants with normal and abnormal liver function during follow-up using the Mann-Whitney U test for continuous variables and the chi-square test for categorical variables. ${ }^{b}$ Percentage was calculated within the same group. ${ }^{c}$ Abnormal liver function was defined as ALT $>33 \mathrm{U} / \mathrm{L}$, AST $>27 \mathrm{U} / \mathrm{L}$, or $\gamma-\mathrm{GT} \geq 39 \mathrm{U} / \mathrm{L}$ for women and $\gamma$-GT $\geq 50 \mathrm{U} / \mathrm{L}$ for men. ${ }^{\mathrm{d}}$ The values of BMI were categorized into underweight $\left(<18.5 \mathrm{~kg} / \mathrm{m}^{2}\right)$, normal weight $\left(18.5-23.9 \mathrm{~kg} / \mathrm{m}^{2}\right)$, overweight $\left(24.0-26.9 \mathrm{~kg} / \mathrm{m}^{2}\right)$, and obese $\left(\geq 27 \mathrm{~kg} / \mathrm{m}^{2}\right)$. 
Table 3. Percentage of variation explained by liver-associated dietary patterns and factor loadings of 22 food items by reduced rank regression analysis.

\begin{tabular}{ccc}
\hline Food Items & Explained Variation (\%) & Factor Loading \\
\hline Soy sauce or other dips & 45.9 & 0.47 \\
Sugar-sweetened beverages & 8.19 & 0.46 \\
Preserved and processed foods & 7.34 & 0.22 \\
Seafood & 0.57 & -0.31 \\
Fruits & 1.43 & -0.30 \\
Eggs & 1.51 & -0.30 \\
Dark-colored vegetables & 1.08 & -0.24 \\
Honey/jam & 0.04 & -0.19 \\
Light-colored vegetables & 0.47 & -0.17 \\
Sweet bread & 7.76 & -0.15 \\
Whole grains & 0.19 & -0.12 \\
Milk & 5.59 & -0.10 \\
Fried rice/flour products & 2.78 & -0.09 \\
Instant noodles & 0.81 & -0.07 \\
Legumes/soy products & 1.14 & -0.07 \\
Root crops & 0.06 & -0.04 \\
Rice/flour products & 0.02 & -0.01 \\
Dairy products & 5.14 & 0.14 \\
Organ meats & 0.25 & 0.14 \\
Meats & 1.19 & 0.07 \\
Vegetables with added oil/fats & $<0.0001$ & 0.07 \\
Deep-fried foods & 0.18 & 0.03 \\
\hline
\end{tabular}

\subsection{Interactive Association of Dietary Pattern, Lifestyles, and Liver Function Biomarkers}

Our study observed the higher odds of experiencing abnormal liver function biomarkers for participants who smoked, drank alcohol, had abnormal sleep duration ( $<6 \mathrm{~h}$ or $>8 \mathrm{~h}$ ), did less physical activity ( $<2 \mathrm{~h} /$ week), and had abnormal BMI status in the fully adjusted model (Supplementary Table S2). However, second-hand smokers and past drinkers had no association with abnormal liver function. We found that participants who smoked or drank on a daily basis were correlated with higher odds of developing liver abnormality by $54 \%(95 \%$ CI: $1.47,1.61)$ and $57 \%(95 \%$ CI: $1.38,1.78)$, respectively, compared to those who were non-smokers or non-drinkers. Moreover, participants who had less physical activity were $25 \%$ more likely to have higher odds of abnormal liver function ( $95 \%$ CI: $1.17,1.33)$ compared to those who had more active physical activity ( $>2 \mathrm{~h} /$ week). Participants with underweight, overweight, or obesity were associated with much higher odds of abnormal liver function, by $71 \%$ (95\% CI: 1.62, 1.80), 215\% (95\% CI: 2.96, 3.39), and $352 \%$ (95\% CI: $4.23,4.85)$, respectively, compared to those with normal weight.

The association of dietary pattern across the quartiles and concordance lifestyle with the risk of abnormal liver function is presented in Table 4. Both higher quartiles of liverassociated dietary patterns and concordance lifestyle were positively correlated with higher odds of abnormal liver function. In model 2, participants in the highest quartile of liver-associated dietary pattern or concordance lifestyle were associated with higher odds of abnormal liver function of $8 \%(95 \%$ CI: $1.04,1.12)$ and $42 \%$ (95\% CI: $1.31,1.53)$, respectively, compared to those in the corresponding reference group. Furthermore, we observed the association of different proportions of dietary or lifestyle exposure with abnormal liver enzymes in both genders (Supplementary Table S3). Male participants in the highest quartile of liver-associated dietary patterns had increased odds of abnormal liver function by $13 \%$ compared to those in the lowest quartile. Female participants in higher quartiles of liver-associated dietary pattern had higher odds of abnormal liver function by $9-18 \%$. Male participants who were past smokers but not current smokers had greater odds of abnormal liver function, while female participants who were past or current smokers had higher odds of abnormal liver function. Male participants who were past or current drinkers had increased odds of abnormal liver function, while female 
participants who were frequent drinkers ( $\geq 3$ times/week) had greater odds of abnormal liver function. Male participants who had long sleep duration had higher odds of abnormal liver function, while female participants who had short or long sleep duration had greater odds of abnormal liver function. Male and female participants who had less physical activity had increased odds of abnormal liver function by $31 \%$ (95\% CI: $1.10,1.55)$ and $24 \%$ (95\% CI: 1.03, 1.48), respectively.

Table 4. Logistic regression models for the association of liver-associated dietary patterns and concordance lifestyle with abnormal liver function $(n=62,645)$.

\begin{tabular}{|c|c|c|c|c|}
\hline \multirow{3}{*}{ Quartile } & \multicolumn{2}{|c|}{ Dietary Pattern } & \multicolumn{2}{|c|}{ Concordance Lifestyle ${ }^{a}$} \\
\hline & 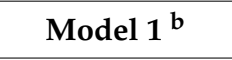 & Model $2^{c}$ & Model $1^{b}$ & Model $2^{c}$ \\
\hline & OR $(95 \%$ CI $)$ & OR $(95 \%$ CI $)$ & OR $(95 \%$ CI $)$ & OR (95\% CI) \\
\hline 1 & \multicolumn{2}{|c|}{1.00 (Ref) } & \multicolumn{2}{|c|}{$1.00(\operatorname{Ref})$} \\
\hline 2 & $1.02(0.99,1.04)$ & $1.03(0.99,1.07)$ & $1.40(1.30,1.50)$ & $1.23(1.14,1.32)$ \\
\hline 3 & $1.06(1.03,1.09)$ & $1.07(1.03,1.11)$ & $1.64(1.53,1.75)$ & $1.29(1.21,1.39)$ \\
\hline 4 & $1.09(1.06,1.13)$ & $1.08(1.04,1.12)$ & $1.83(1.70,1.96)$ & $1.42(1.31,1.53)$ \\
\hline$p$ for Trend & $<0.001$ & 0.001 & $<0.001$ & $<0.001$ \\
\hline
\end{tabular}

a Concordance lifestyle scores were calculated by computing all lifestyle variables and categorizing into quartiles

${ }^{b}$ Model 1 was adjusted for age and gender. ${ }^{c}$ For dietary pattern, model 2 was adjusted for age, gender, marital status, education, family income, body mass index, body fat, systolic pressure, diastolic pressure, smoking status, drinking status, sleep duration, physical activity, and iron biomarkers. For concordance lifestyle, model 2 was adjusted for age, gender, marital status, education, family income, body mass index, body fat, systolic pressure, diastolic pressure, dietary categories, and iron biomarkers.

Moreover, the interaction between liver-associated dietary patterns and concordance lifestyle for the risk of abnormal liver function is shown in Table 5. Individuals who were in both the highest quartile of liver-associated dietary patterns and concordance lifestyle were more likely to have increased odds of developing liver function abnormality, by $114 \%$ (95\% CI: 2.02, 2.26), compared to those who were in both the lowest quartile of liverassociated dietary pattern and concordance lifestyle.

Table 5. Adjusted interaction models between dietary pattern and concordance lifestyle across the quartiles for the risk of abnormal liver function $(n=62,645)^{\mathrm{a}}$.

\begin{tabular}{ccccc}
\hline \multirow{2}{*}{$\begin{array}{c}\text { Quartiles of } \\
\text { Dietary Pattern }\end{array}$} & $\mathbf{4}$ Quartiles of Concordance Lifestyle \\
\cline { 2 - 5 } & OR (95\% CI) & OR (95\% CI) & OR (95\% CI) & OR (95\% CI) \\
\cline { 2 - 5 } & $1.00($ Ref) & $1.25(1.18,1.32)$ & $1.53(1.46,1.61)$ & $2.10(1.99,2.21)$ \\
\hline 1 & $1.08(1.02,1.14)$ & $1.26(1.19,1.33)$ & $1.56(1.48,1.65)$ & $2.10(1.99,2.22)$ \\
2 & $1.09(1.03,1.15)$ & $1.28(1.21,1.36)$ & $1.61(1.53,1.70)$ & $2.11(1.98,2.23)$ \\
4 & $1.21(1.14,1.28)$ & $1.33(1.26,1.41)$ & $1.63(1.55,1.72)$ & $2.14(2.02,2.26)$ \\
\hline
\end{tabular}

a Adjusted model for age, gender, marital status, education, family income, body mass index, body fat, systolic pressure, diastolic pressure, and iron biomarkers.

\section{Discussion}

Our study generated interactive models and determined the effects of joint exposure of liver-associated dietary patterns and concordance lifestyle on developing abnormal liver function. This study revealed that individuals with poor lifestyle behaviors such as smoking, alcohol consumption, abnormal sleep duration, and less physical activity were associated with higher odds of developing abnormal liver function. These associations were independent of age, gender, marital status, education, family income, BMI, body fat, blood pressure, and iron biomarkers. Correspondingly, participants who were in higher quartiles of the reduced rank regression-derived dietary pattern, namely the liver-associated dietary pattern, were significantly associated with disease progression. 
Interestingly, higher quartiles of dietary pattern and lifestyle interaction were prone to liver dysfunction. To our knowledge, this study provided the first evidence that the interplay of poor dietary pattern and lifestyle behaviors might worsen liver function outcomes in a longitudinal setting.

Previous studies showed a positive correlation of poor lifestyle behaviors with liver dysfunction [14,27-29]. Accordingly, our investigation revealed that individuals who were exposed to poor lifestyle behaviors were associated with abnormal liver function biomarkers, which indicated a higher risk of developing liver dysfunction. The liverassociated dietary pattern showed a correlation with higher odds of developing abnormal liver function. The characteristics of the derived dietary pattern, with high consumption of dipping sauces, sugar-sweetened beverages, and processed foods but low consumption of presumably healthy food, such as seafood, fruits, eggs, and dark vegetables, were similar to an unhealthy dietary pattern and could be associated with an increased risk of developing abnormal liver function [30]. A previous investigation among the Taiwanese population indicated that Western dietary patterns, known as unhealthy diets, appeared to be a risk factor in developing abnormal serum ALT levels in female participants but not in male participants [11]. In gender-specific analyses, we observed that the role of dietary patterns on disease progression occurred across quartiles in female participants, while only the highest quartile of dietary pattern showed a significant association with abnormal liver function in male participants. Previous evidence suggests that an unhealthy dietary pattern, mostly consisting of high-fat and high-energy foods, is closely related to liver disease; however, only a few studies have examined dietary patterns with greater adherence to refined foods and sugary drinks or high-energy but low-nutrient-density foods in NAFLD progression [31,32]. Interestingly, we found a significant correlation between the development of liver abnormality and the dietary pattern characterized by high sodium foods and sweetened beverages. Dipping sauces and processed foods consumed in the liver-associated dietary pattern contain high sodium, which might be related to the development of liver disease [30,33]. In a cross-sectional study among 100,177 participants in Korea, the dietary pattern with high sodium intake, including sauces and processed meals, was significantly associated with a greater prevalence of NAFLD ( $\mathrm{PR}=1.25,95 \% \mathrm{CI}: 1.18,1.32$ in men and PR $=1.32,95 \% \mathrm{CI}$ : 1.18, 1.47 in women) [30]. In addition, a cross-sectional study among NAFLD patients demonstrated a positive association between the dietary pattern characterized by refined grains, fried foods, meat, and processed meat and the presence of NAFLD [33]. Despite the fact that the mechanism for the effect of sodium on liver disease is not completely understood, an experimental study conducted in rats suggested that sodium could have a direct effect on obesity through inducing the hypertrophy of adipocytes by improving insulin sensitivity and glucose metabolism stimulated by insulin in the adipocytes [34]. In agreement with our study, the results revealed that overweight and obesity were positively correlated with a greater increased risk of abnormal liver function.

Moreover, individuals who adhered to fast foods or sugar sweetened beverages (3-5 times/week) were more likely to increase serum ALT (OR = 1.27, 95\% CI: 1.05, 1.54 and $\mathrm{OR}=1.24,95 \% \mathrm{CI}: 1.07,1.42$, respectively) compared to those who did not consume these foods among Korean overweight or obese teenage students [35]. Additionally, a randomized intervention study among 47 overweight or obese subjects aged 20-50 years suggested that subjects who consumed $1 \mathrm{~L}$ of sugar-containing drinks daily for 6 months significantly increased liver and visceral fat [36]. A systematic review and meta-analysis of seven studies (six cross-sectional studies and one cohort study) involving 4639 participants reported that sugar-sweetened beverage consumers were more likely to be correlated with an increased risk of developing NAFLD of $53 \%\left(R R=1.53,95 \%\right.$ CI: $\left.1.34,1.75, I^{2}=0 \%\right)$ compared to non-consumers [37]. Some reports suggest that sweeteners such as fructose and sucrose in soft drinks and snacks could stimulate lipogenesis and inhibit mitochondrial $\beta$-oxidation of fatty acids, which leads to increases in liver fat and serum liver enzyme levels [32,38]. Nutrient components of the healthy dietary pattern, such as dietary fiber, 
vitamins, and minerals, were not abundant in our derived liver-associated dietary pattern, which could plausibly suggest that an increased risk of developing liver abnormality was found among the participants following the liver-associated dietary pattern.

Regarding the association of smoking and abnormal liver function, a review study among 20,149 individuals aged above 18 years supported a positive relationship between smoking and NAFLD (pooled OR $=1.110,95 \%$ CI: 1.028, 1.199) [14]. In addition, a population-based study in China demonstrated higher odds for NAFLD among individuals who were former smokers (OR $=1.45,95 \%$ CI: 1.05, 2.00) or current heavy smokers $(\mathrm{OR}=2.29,95 \% \mathrm{CI}: 1.30,4.03)$ compared to non-smokers [39]. Moreover, previous studies have also observed the independent and combined effects of active cigarette smoking and alcohol consumption on an elevation of serum liver enzyme levels [40,41]. An epidemiological cohort study among middle-aged and elderly subjects in Germany showed that individuals who were combined heavy smokers ( $\geq 21$ cigarettes/d) and moderate to heavy drinkers ( $\geq 100 \mathrm{~g}$ alcohol/week) were associated with an increased odds of elevated $\gamma$-GT to 2.9 (95\% CI: 1.1, 7.6) and to 3.8 (95\% CI: 2.2, 6.6) in women and men, respectively, compared to non-smokers and non-drinkers [40]. Another study also supported this finding: a population-based prospective cohort study conducted among 5946 adults in rural and urban areas of South Korea observed that combined heavy cigarette smoking ( $>20$ pack years) and heavy alcohol consumption $(\geq 24 \mathrm{~g} / \mathrm{d}$ ) showed a supra-additive effect with synergic index (SI) $>1$ on an elevation of $\gamma$-GT (SI $=2.03,95 \%$ CI: 1.75, 2.35) and AST levels (SI $=4.55,95 \%$ CI: 3.12, 6.61) [41]. This association could be addressed by the assumption that both smoking and alcohol consumption might stimulate oxidative stress and induce the depletion of anti-inflammatory substances such as glutathione in various tissues, including the liver [40,42]. Likewise, a rat study revealed that oxidative stress in the liver induced by chronic combined administration of alcohol and nicotine could lead to additive oxidative damage, such as lipid peroxidation in the liver [42].

The association between sleep duration and an increased risk of liver abnormalities has been widely investigated in epidemiologic studies, although the results remain inconclusive. Interestingly, our study reported that both short and long sleep duration played a role in developing liver abnormalities. A meta-analysis study including 59,094 participants found an increased risk of having NAFLD among participants who had short sleep duration compared to those who had longer sleep duration (pooled RR $=1.19,95 \%$ CI: 1.04 , $1.36, I^{2}=0 \%$ [ [43]. A population-based longitudinal study among 12,306 Japanese revealed that short sleep duration $\leq 5 \mathrm{~h}$ in both women (HR $=1.46,95 \% \mathrm{CI}: 1.05,2.04)$ and men $(\mathrm{HR}=1.39,95 \%$ CI: $1.13,1.72)$ was significantly correlated with an increased risk of developing NAFLD compared to longer sleep duration ( $>7 \mathrm{~h}$ ) after follow-up for 6.8 (for men) or 7 (for women) years [44]. Previous reports also suggest that dietary intake might have a mediating effect on the association between sleep duration and liver abnormalities [45,46]. Thus, a link between short sleep duration $(\leq 6 \mathrm{~h})$ and greater calorie intake has been observed as compared to normal sleep duration ( $7 \mathrm{~h}$ or 7-8 h) [45,46], and individuals who had short sleep duration (5-6 h) had a tendency toward increased carbohydrate, sugar, and fat consumption compared to those who had normal sleep duration (7-8 h) [46]. As a result, short sleep duration could be accompanied by changes in appetite-related hormones, eating behavior, and nutrient metabolism, which might further raise the risk of certain chronic diseases such as liver disease [47].

Evidence supporting our findings has shown that less physical activity was associated with aberrant liver function. A cross-sectional study conducted among 139,056 middleaged Koreans reported an inverse association between physical activity and NAFLD (PR $=0.80-0.94,95 \%$ CI: 0.78-0.92, 0.82-0.95) [48]. Furthermore, Ryu et al. [48] also found that participants who were physically inactive tended to have unhealthy dietary patterns. Subjects with prolonged sitting times were more likely to be associated with an increased calorie intake, by $36 \%(95 \%$ CI: 1.31, 1.43) [48]. This finding was consistent with the results from a small-scale cross-sectional study of 74 British adults, which found that NAFLD individuals had approximately an additional half-hour per day of sedentary 
behavior $(1318 \pm 68$ vs. $1289 \pm 60 \mathrm{~min} /$ day, $p<0.05)$ and walked fewer steps $(8483 \pm 2926$ vs. $10,377 \pm 3529$ steps/day, $p<0.01$ ) compared to the healthy controls [29]. Adopting sedentary behaviors over a long period of time could limit overall energy expenditure by muscle contraction and impair lipoprotein lipase activity, which leads to the inhibition of triglyceride uptake by skeletal muscles and impedes fat metabolism in peripheral tissues; thus, the role of sedentary behavior might indirectly affect fat metabolism in the liver and further be related to the etiology of NAFLD [29,49].

With respect to the significant association between concordance lifestyle behavior and abnormal liver function, we found that joint lifestyle behaviors played a more significant role in the presence of abnormal liver function than dietary pattern did. This finding could be explained by the fact that combined lifestyle habits can have more significant effects on improving or worsening the disease of interest [50]. A previous study demonstrated that an unhealthy dietary pattern was correlated with a higher risk of NAFLD, especially among obese participants [51]. Poor lifestyle habits, such as a lack of exercise, exacerbated abnormal liver function, independently of body weight. As matter of fact, our participants were more likely to have normal weight status, and this could explain the limited association of the dietary pattern with the development of liver abnormalities.

A major strength of our study was the large sample size, providing a reliable statistical power to evaluate the longitudinal data. Because liver disease is caused by multifactorial exposure [52], we expanded our analysis to observe the combined exposures of dietary pattern and lifestyle factors in the development of disease outcomes. The novelty of exploring the synergistic correlation between four-component evolutionary concordance lifestyle and dietary pattern-specific liver abnormality was also our notable strength. Moreover, our study was the first study to conduct such an interaction analysis in Taiwan. As a result, our findings might make a significant contribution to elucidate the effect of various lifestyle variables on the prevention and management of liver abnormalities. However, there are some limitations that should be mentioned. First, the histological definition of liver disease, such as through biopsy, was not included because invasive procedures were not acceptable in a population-based epidemiological investigation [53]. Therefore, we described the study participants with normal or abnormal liver function according to serum liver enzyme levels such as ALT, AST, and $\gamma$-GT [5]. Second, because lifestyle habits, including smoking, alcohol drinking, sleeping habits, and physical activity, were self-reported, misclassification was inevitable. Third, the well-known limitations of SQ-FFQ, including recall errors, a limited number of food items, and difficulties in estimating intake frequencies, were also present in this study [54].

\section{Conclusions}

Our findings suggest that individuals who followed the liver-associated dietary pattern alone or combined with a concordance lifestyle are positively associated with abnormal liver function among adults in Taiwan. Despite these findings of epidemiological investigation, the prospective cohort study of the interaction between several lifestyle behaviors in the development of liver disease is required.

Supplementary Materials: The following are available online at https:/ /www.mdpi.com/article/10 .3390 / nu13103591/s1, Table S1: Multivariate linear regression of liver function biomarkers across the quartiles of dietary pattern, Table S2: Logistic regression models for the association of lifestyle variables with abnormal liver function biomarkers $(n=62,645)$. Table S3: Multiple logistic regression for the association of dietary pattern or lifestyle variables with abnormal liver function biomarkers in both genders $(n=62,645)$.

Author Contributions: Conceptualization, R.P. and J.C.-J.C.; data curation, C.-Y.H., Y.-K.C. and H.-A.L.; formal analysis, R.P. and B.S.W.; writing-original draft preparation, R.P. and J.C.-J.C.; writing-review and editing, J.C.-J.C.; supervising, C.-Y.H., Y.-K.C. and J.C.-J.C. All authors have read and agreed to the published version of the manuscript.

Funding: This research received no external funding. 
Institutional Review Board Statement: The study was approved by the Taipei Medical UniversityJoint Institutional Review Board (TMU-JIRB) no. 202001055, approved on 11 November 2020.

Informed Consent Statement: All subjects signed the consent form authorized by the Mei Jau Health Management Institution.

Data Availability Statement: Data were obtained from the Mei Jau Health Management Institution, but were restricted for research use only. The data are not publicly available.

Acknowledgments: We gratefully acknowledge the Mei Jau Health Management Institution for collecting and providing their data for the present study.

Conflicts of Interest: The authors declare no conflict of interest.

\section{References}

1. Tsai, P.-Y.; Yen, C.-J.; Li, Y.-C.; Chiu, T.-Y.; Chen, C.-Y.; Jan, C.-F. Association between abnormal liver function and risk factors for metabolic syndrome among freshmen. J. Adolesc. Health 2007, 41, 132-137. [CrossRef]

2. Chalasani, N.; Younossi, Z.; Lavine, J.E.; Diehl, A.M.; Brunt, E.M.; Cusi, K.; Charlton, M.; Sanyal, A.J. The diagnosis and management of non-alcoholic fatty liver disease: Practice guideline by the American Association for the Study of Liver Diseases, American College of Gastroenterology, and the American Gastroenterological Association. Hepatology 2012, 55, $2005-2023$. [CrossRef]

3. Moshayedi, H.; Ahrabi, R.; Mardani, A.; Sadigetegad, S.; Farhudi, M. Association between non-alcoholic fatty liver disease and ischemic stroke. Iran. J. Neurol. 2014, 13, 144-148.

4. Schindhelm, R.K.; Dekker, J.M.; Nijpels, G.; Bouter, L.M.; Stehouwer, C.D.; Heine, R.J.; Diamant, M. Alanine aminotransferase predicts coronary heart disease events: A 10-year follow-up of the Hoorn Study. Atherosclerosis 2007, 191, 391-396. [CrossRef] [PubMed]

5. Ahmed, Z.; Ahmed, U.; Walayat, S.; Ren, J.; Martin, D.K.; Moole, H.; Koppe, S.; Yong, S.; Dhillon, S. Liver function tests in identifying patients with liver disease. Clin. Exp. Gastroenterol. 2018, 11, 301-307. [CrossRef] [PubMed]

6. Alexander, K.S.; Zakai, N.A.; Lidofsky, S.D.; Callas, P.W.; Judd, S.E.; Tracy, R.P.; Cushman, M. Non-alcoholic fatty liver disease, liver biomarkers and stroke risk: The reasons for geographic and racial differences in stroke cohort. PLoS ONE 2018, 13, e0194153. [CrossRef]

7. Hsieh, M.-H.; Lin, W.-Y.; Chien, H.-H.; Chien, L.-H.; Huang, C.-K.; Yang, J.-F.; Chang, N.-C.; Huang, C.-F.; Wang, C.-L.; Chuang, W.-L.; et al. Waist circumference, body mass index, serum uric acid, blood sugar, and triglyceride levels are important risk factors for abnormal liver function tests in the Taiwanese population. Kaohsiung J. Med. Sci. 2012, 28, 470-476. [CrossRef] [PubMed]

8. Younossi, Z.; Anstee, Q.M.; Marietti, M.; Hardy, T.; Henry, L.; Eslam, M.; George, J.; Bugianesi, E. Global burden of NAFLD and NASH: Trends, predictions, risk factors and prevention. Nat. Rev. Gastroenterol. Hepatol. 2018, 15, 11-20. [CrossRef] [PubMed]

9. Estes, C.; Chan, H.L.Y.; Chien, R.N.; Chuang, W.-L.; Fung, J.; Goh, G.B.-B.; Hu, T.H.; Huang, J.-F.; Jang, B.K.; Jun, D.W.; et al. Modelling NAFLD disease burden in four Asian regions-2019-2030. Aliment. Pharmacol. Ther. 2020, 51, 801-811. [CrossRef]

10. Chen, C.-H.; Huang, M.-H.; Yang, J.-C.; Nien, C.-K.; Yang, C.-C.; Yeh, Y.-H.; Yueh, S.-K. Prevalence and risk factors of nonalcoholic fatty liver disease in an adult population of Taiwan: Metabolic significance of nonalcoholic fatty liver disease in nonobese adults. J. Clin. Gastroenterol. 2006, 40, 745-752. [CrossRef]

11. Lin, L.-Y.; Hsu, C.-Y.; Chiou, H.-Y.; Lee, H.-A.; Hsu, L.-M.; Chang, P.-Y.; Kurniawan, A.L.; Chao, J.C.-J. Association between dietary patterns and serum hepatic enzyme levels in adults with dyslipidemia and impaired fasting plasma glucose. Nutrients 2021, 13, 987. [CrossRef]

12. Salehi-sahlabadi, A.; Sadat, S.; Beigrezaei, S.; Pourmasomi, M.; Feizi, A.; Ghiasvand, R.; Hadi, A.; Clark, C.C.T.; Miraghajani, M. Dietary patterns and risk of non-alcoholic fatty liver disease. BMC Gastroenetrol. 2021, 21, 41. [CrossRef]

13. Zelber-Sagi, S.; Nitzan-Kaluski, D.; Goldsmith, R.; Webb, M.; Zvibel, I.; Goldiner, I.; Blendis, L.; Halpern, Z.; Oren, R. Role of leisure-time physical activity in nonalcoholic fatty liver disease: A population-based study. Hepatology 2008, 48, 1791-1798. [CrossRef] [PubMed]

14. Rezayat, A.A.; Moghadam, M.D.; Nour, M.G.; Shirazinia, M.; Ghodsi, H.; Zahmatkesh, M.R.R.; Noghabi, M.T.; Hoseini, B.; Rezayat, K.A. Association between smoking and non-alcoholic fatty liver disease: A systematic review and meta-analysis. SAGE Open Med. 2018, 6, 2050312117745223. [CrossRef]

15. Muga, M.A.; Owili, P.O.; Hsu, C.-Y.; Rau, H.-H.; Chao, J.C.-J. Association between dietary patterns and cardiovascular risk factors among middle-aged and elderly adults in Taiwan: A population-based study from 2003 to 2012. PLoS ONE 2016, 11, e0157745. [CrossRef]

16. Liu, C.-Y.; Chou, Y.-C.; Chao, J.C.-J.; Hsu, C.-Y.; Cha, T.-L.; Tsao, C.-W. The association between dietary patterns and semen quality in a general asian population of 7282 males. PLOS ONE 2015, 10, e0134224. [CrossRef]

17. Paramastri, R.; Hsu, C.-Y.; Lee, H.-A.; Lin, L.-Y.; Kurniawan, A.L.; Chao, J.C.-J. Association between dietary pattern, lifestyle, anthropometric status, and anemia-related biomarkers among adults: A population-based study from 2001 to 2015 . Int. J. Environ. Res. 2021, 18, 3438. [CrossRef] 
18. Chin, C.-C.; Kuo, Y.-H.; Yeh, C.-Y.; Chen, J.-S.; Tang, R.; Changchien, C.-R.; Wang, J.-Y.; Huang, W.-S. Role of body mass index in colon cancer patients in Taiwan. World J. Gastroenterol. 2012, 18, 4191-4198. [CrossRef]

19. MJ Group. MJ Health Screening Center Questionnaire QR-121-1 MJ2011.06-1104TW. Available online: https://www.mjlife.com/ index.aspx?lang=chi\&fn=index (accessed on 5 April 2021).

20. Meyer, K.A.; Sijtsma, F.P.; Nettleton, J.A.; Steffen, L.M.; Van Horn, L.; Shikany, J.M.; Gross, M.D.; Mursu, J.; Traber, M.G.; Jacobs, D.R., Jr. Dietary patterns are associated with plasma $\mathrm{F}_{2}$-isoprostanes in an observational cohort study of adults. Free Radic. Biol. Med. 2013, 57, 201-209. [CrossRef]

21. Hoffmann, K.; Schulze, M.B.; Schienkiewitz, A.; Nöthlings, U.; Boeing, H. Application of a new statistical method to derive dietary patterns in nutritional epidemiology. Am. J. Epidemiol. 2004, 159, 935-944. [CrossRef]

22. Kurniawan, A.L.; Hsu, C.-Y.; Lee, H.-A.; Rau, H.-H.; Paramastri, R.; Syauqy, A.; Chao, J.C.-J. Comparing two methods for deriving dietary patterns associated with risk of metabolic syndrome among middle-aged and elderly Taiwanese adults with impaired kidney function. BMC Med. Res. Methodol. 2020, 20, 1-12. [CrossRef] [PubMed]

23. Batis, C.; Mendez, M.A.; Gordon-Larsen, P.; Sotres-Alvarez, D.; Adair, L.; Popkin, B. Using both principal component analysis and reduced rank regression to study dietary patterns and diabetes in Chinese adults. Public Health Nutr. 2016, 19, 195-203. [CrossRef] [PubMed]

24. Frank, J.; Massey, J.R. The Kolmogorov-Smirnov test for goodness of fit. J. Am. Stat. Assoc. 1951, 253, 68-78.

25. Jonquet, C.; Cerri, S. The Strobe model: Dynamic service generation on the grid. Appl. Artif. Intell. 2005, 19, 967-1013. [CrossRef]

26. Uyanık, G.K.; Güler, N. A study on multiple linear regression analysis. Procedia Soc. Behav. Sci. 2013, 106, 234-240. [CrossRef]

27. Kim, C.-W.; Yun, K.E.; Jung, H.-S.; Chang, Y.; Choi, E.-S.; Kwon, M.-J.; Lee, E.-H.; Woo, E.J.; Kim, N.H.; Shin, H.; et al. Sleep duration and quality in relation to non-alcoholic fatty liver disease in middle-aged workers and their spouses. J. Hepatol. 2013, 59, 351-357. [CrossRef]

28. Marin-Alejandre, B.A.; Abete, I.; Cantero, I.; Riezu-Boj, J.I.; Milagro, F.I.; Monreal, J.I.; Elorz, M.; Herrero, J.I.; Benito-Boillos, A.; Quiroga, J.; et al. Association between sleep disturbances and liver status in obese subjects with nonalcoholic fatty liver disease: A comparison with healthy controls. Nutrients 2019, 11, 322. [CrossRef] [PubMed]

29. Hallsworth, K.; Thoma, C.; Moore, S.; Ploetz, T.; Anstee, Q.M.; Taylor, R.; Day, C.P.; Trenell, M.I. Non-alcoholic fatty liver disease is associated with higher levels of objectively measured sedentary behaviour and lower levels of physical activity than matched healthy controls. Frontline Gastroenterol. 2015, 6, 44-51. [CrossRef]

30. Choi, Y.; Lee, J.E.; Chang, Y.; Kim, M.K.; Sung, E.; Shin, H.; Ryu, S. Dietary sodium and potassium intake in relation to non-alcoholic fatty liver disease. Br. J. Nutr. 2016, 116, 1447-1456. [CrossRef]

31. Mirmiran, P.; Gaeini, Z.; Bahadoran, Z.; Azizi, F. Elevated serum levels of aminotransferases in relation to unhealthy foods intake: Tehran lipid and glucose study. BMC Endocr. Disord. 2019, 19, 100. [CrossRef]

32. Tapsell, L.C.; Neale, E.P.; Satija, A.; Hu, F.B. Foods, nutrients, and dietary patterns: Interconnections and implications for dietary guidelines. Adv. Nutr. 2016, 7, 445-454. [CrossRef] [PubMed]

33. Shim, P.; Choi, D.; Park, Y. Association of blood fatty acid composition and dietary pattern with the risk of non-alcoholic fatty liver disease in patients who underwent cholecystectomy. Ann. Nutr. Metab. 2017, 70, 303-311. [CrossRef]

34. Fonseca-Alaniz, M.H.; Takada, J.; Andreotti, S.; De Campos, T.B.; Campaña, A.B.; Borges-Silva, C.N.; Lima, F.B. High sodium intake enhances insulin-stimulated glucose uptake in rat epididymal adipose tissue. Obesity 2008, 16, 1186-1192. [CrossRef]

35. Lee, E.-Y.; Choi, H.Y.; Cho, H.; Kim, B.H.; Ki, M. Health behavior associated with liver enzymes among obese Korean adolescents, 2009-2014. PLoS ONE 2018, 13, e0190535. [CrossRef]

36. Maersk, M.; Belza, A.; Stødkilde-Jørgensen, H.; Ringgaard, S.; Chabanova, E.; Thomsen, H.; Pedersen, S.B.; Astrup, A.; Richelsen, B. Sucrose-sweetened beverages increase fat storage in the liver, muscle, and visceral fat depot: A 6-mo randomized intervention study. Am. J. Clin. Nutr. 2011, 95, 283-289. [CrossRef]

37. Wijarnpreecha, K.; Thongprayoon, C.; Edmonds, P.; Cheungpasitporn, W. Associations of sugar-and artificially sweetened soda with nonalcoholic fatty liver disease: A systematic review and meta-analysis. QJM Int. J. Med. 2016, 109, 461-466. [CrossRef] [PubMed]

38. Softic, S.; Cohen, D.E.; Kahn, C.R. Role of dietary fructose and hepatic de novo lipogenesis in fatty liver disease. Dig. Dis. Sci. 2016, 61, 1282-1293. [CrossRef]

39. Liu, Y.; Dai, M.; Bi, Y.; Xu, M.; Xu, Y.; Li, M.; Wang, T.; Huang, F.; Xu, B.; Zhang, J. Active smoking, passive smoking, and risk of nonalcoholic fatty liver disease (NAFLD): A population-based study in China. J. Epidemiol. 2013, 23, 115-121. [CrossRef] [PubMed]

40. Breitling, L.P.; Raum, E.; Müller, H.; Rothenbacher, D.; Brenner, H. Synergism between smoking and alcohol consumption with respect to serum gamma-glutamyltransferase. Hepatology 2009, 49, 802-808. [CrossRef]

41. Park, E.Y.; Lim, M.K.; Oh, J.-K.; Cho, H.; Bae, M.J.; Yun, E.H.; Kim, D.-I.; Shin, H.-R. Independent and supra-additive effects of alcohol consumption, cigarette smoking, and metabolic syndrome on the elevation of serum liver enzyme levels. PLoS ONE 2013, 8, e63439. [CrossRef]

42. Husain, K.; Scott, B.R.; Reddy, S.K.; Somani, S.M. Chronic ethanol and nicotine interaction on rat tissue antioxidant defense system. Alcohol 2001, 25, 89-97. [CrossRef]

43. Wijarnpreecha, K.; Thongprayoon, C.; Panjawatanan, P.; Ungprasert, P. Short sleep duration and risk of nonalcoholic fatty liver disease: A systematic review and meta-analysis. J. Gastroenterol. Hepatol. 2016, 31, 1802-1807. [CrossRef] 
44. Okamura, T.; Hashimoto, Y.; Hamaguchi, M.; Obora, A.; Kojima, T.; Fukui, M. Short sleep duration is a risk of incident nonalcoholic fatty liver disease: A population-based longitudinal study. J. Gastrointest. Liver Dis. 2019, 28, 73-81. [CrossRef]

45. Stern, J.H.; Grant, A.S.; Thomson, C.A.; Tinker, L.; Hale, L.; Brennan, K.M.; Woods, N.F.; Chen, Z. Short sleep duration is associated with decreased serum leptin, increased energy intake and decreased diet quality in postmenopausal women. Obesity 2014, 22, E55-E61. [CrossRef]

46. Grandner, M.A.; Jackson, N.; Gerstner, J.R.; Knutson, K.L. Dietary nutrients associated with short and long sleep duration. Data from a nationally representative sample. Appetite 2013, 64, 71-80. [CrossRef]

47. Dashti, H.S.; Scheer, F.A.; Jacques, P.F.; Lamon-Fava, S.; Ordovás, J.M. Short sleep duration and dietary intake: Epidemiologic evidence, mechanisms, and health implications. Adv. Nutr. 2015, 6, 648-659. [CrossRef]

48. Ryu, S.; Chang, Y.; Jung, H.-S.; Yun, K.E.; Kwon, M.-J.; Choi, Y.; Kim, C.-W.; Cho, J.; Suh, B.-S.; Cho, Y.K.; et al. Relationship of sitting time and physical activity with non-alcoholic fatty liver disease. J. Hepatol. 2015, 63, 1229-1237. [CrossRef] [PubMed]

49. Hamilton, M.T.; Hamilton, D.G.; Zderic, T.W. Role of low energy expenditure and sitting in obesity, metabolic syndrome, type 2 diabetes, and cardiovascular disease. Diabetes 2007, 56, 2655-2667. [CrossRef]

50. Trovato, F.M.; Martines, G.F.; Brischetto, D.; Trovato, G.; Catalano, D. Neglected features of lifestyle: Their relevance in nonalcoholic fatty liver disease. World J. Hepatol. 2016, 8, 1459-1465. [CrossRef] [PubMed]

51. Oddy, W.H.; Herbison, C.E.; Jacoby, P.; Ambrosini, G.L.; O’Sullivan, T.A.; Ayonrinde, O.T.; Olynyk, J.K.; Black, L.J.; Beilin, L.J.; Mori, T.A.; et al. The Western dietary pattern is prospectively associated with nonalcoholic fatty liver disease in adolescene. Am. J. Gastroenterol. 2013, 108, 778-785. [CrossRef] [PubMed]

52. Kalafati, I.-P.; Borsa, D.; Dimitriou, M.; Revenas, K.; Kokkinos, A.; Dedoussis, G.V. Dietary patterns and non-alcoholic fatty liver disease in a Greek case-control study. Nutrition 2019, 61, 105-110. [CrossRef] [PubMed]

53. Joy, D.; Thava, V.R.; Scott, B.B. Diagnosis of fatty liver disease: Is biopsy necessary? Eur. J. Gastroenterol. Hepatol. 2003, 15, 539-543. [CrossRef] [PubMed]

54. Cheng, E.; Um, C.Y.; Prizment, A.E.; Lazovich, D.; Bostick, R.M. Evolutionary-concordance lifestyle and diet and Mediterranean diet pattern scores and risk of incident colorectal cancer in Iowa women. Cancer Epidemiol. Biomark. Prev. 2018, 27, 1195-1202. [CrossRef] [PubMed] 